LEITERS

\section{Functional characterization of desmin mutant p.P419S}

European Journal of Human Genetics (2013) 21, 589-590; doi:10.1038/ejhg.2012.212; published online 3 October 2012

Recently, Hedberg et al ${ }^{1}$ identified a DES mutation (p.P419S) in a Swedish family, suffering from myofibrillar myopathy (MFM) in combination with arrhythmogenic right ventricular cardiomyopathy (ARVC), by next-generation sequencing. Originally, a linkage analysis indicated that the genetic defect is located on chromosome 10q22.3 in this family. ${ }^{2}$ The analysis of muscle biopsies of affected patients demonstrated an aggregation of desmin and further proteins.

The same desmin mutation (p.P419S) was identified before by Olivé et $a l^{3}$ in patients suffering from skeletal myopathy or hypertrophic cardiomyopathy, respectively. However, Hedberg et al ${ }^{1}$ demonstrated that this mutation did not completely co-segregate within the Swedish family, raising the questions on pathogenesis or penetrance, respectively. Of note, in both studies the desmin mutant p.P419S was not functionally characterized. Hence, it is currently difficult to judge the pathological potential of this variant. Especially, it is unclear whether the DES mutation p.P419S is a sufficient molecular trigger for aggregate formation.

For this reason, we introduced this mutation by site-directed mutagenesis into a desmin construct (pEYFP-N1-Desmin) using appropriate oligonucleotides and transfected $\mathrm{H} 9 \mathrm{c} 2, \mathrm{C} 2 \mathrm{C} 12$ and SW-13 cells with mutant and wild-type desmin-eYFP constructs. The filament or aggregate formation was investigated in cell culture
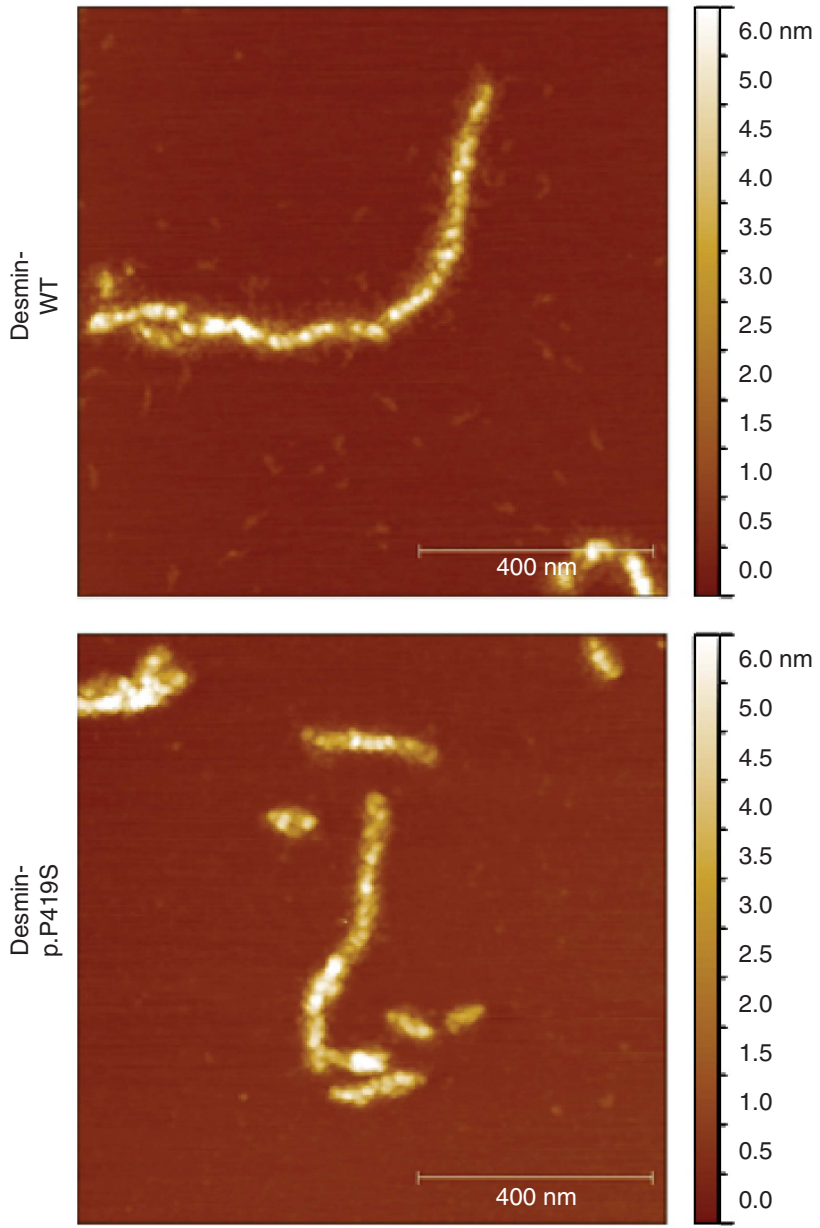

Figure 2 In vitro filament formation of wild-type and mutant (p.P419S) desmin. The desmin molecules were expressed in Escherichia coli and purified by ion-exchange and affinity chromatography. Filament assembly was initiated by the addition of sodium chloride $(100 \mathrm{~mm})$ and was analysed by AFM. The height is colour coded in the representative topography images.
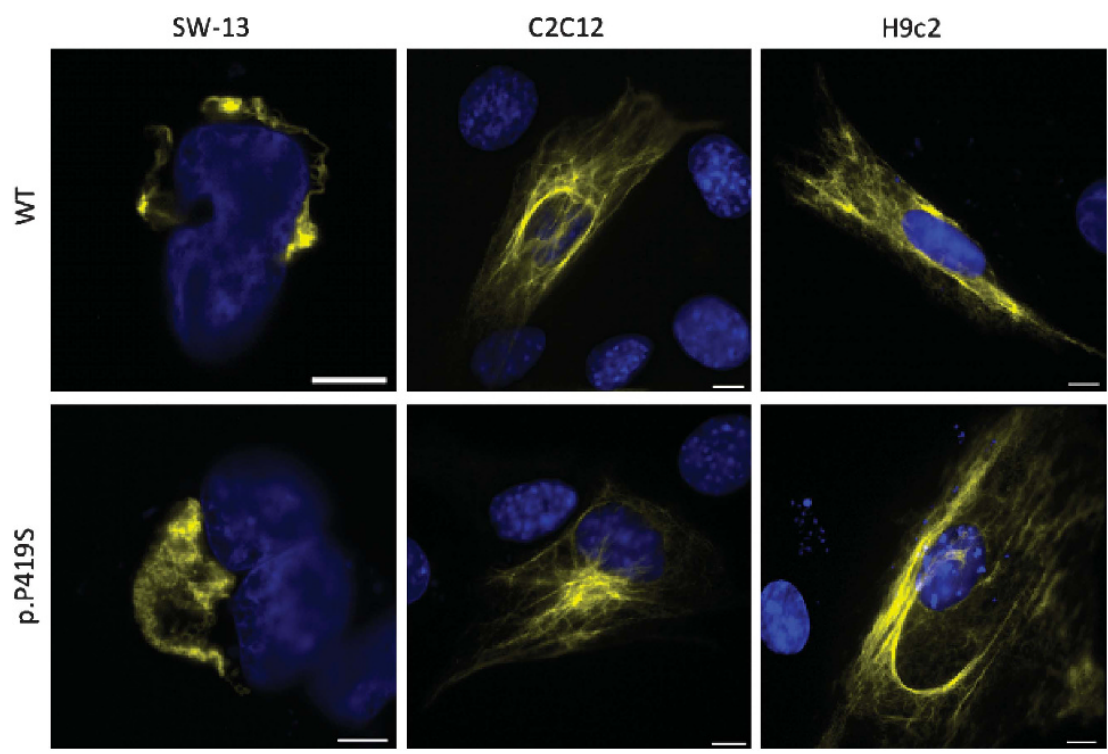

Figure 1 Filament formation of wild-type and mutant (p.P419S) desmin in transfected cells. Representative fluorescence images of transfected SW-13, C2C12 and H9c2 cells expressing desmin-eYFP constructs (yellow) were shown. The nuclei were stained with DAPI. Scale bars represent $10 \mu \mathrm{m}$. 
and the filament formation of purified recombinant mutant desmin was analysed in vitro by atomic force microscopy (AFM), as previously described. ${ }^{4}$

To our surprise, the expression of desmin-p.P419S does not induce an aggregation in either cell line as recently described for other ARVCrelated desmin mutants ${ }^{4-6}$ (Figure 1). The cell culture data were also supported by the AFM analysis virtually yielding undistinguishable desmin filaments between wild-type and desmin-p.P419S in vitro (Figure 2). Thus, our data reveal that the desmin mutant p.P419S published by Hedberg et $a l^{1}$ forms filaments in vitro and in transfected cells. Consequently, it might be important to look for further molecular triggers, which induce or influence the protein aggregation in the Swedish patients suffering from MFM/ARVC. From our point of view, the next-generation sequencing data of Hedberg et al ${ }^{1}$ might provide an important basis for further studies, identifying modifier genes or other molecular abnormalities responsible for desmin aggregate formation.

\section{CONFLICT OF INTEREST}

The authors declare no conflict of interest.

Andreas Brodehl ${ }^{1,4}$, Mareike Dieding ${ }^{2,4}$, Hamdin Cakar ${ }^{3}$, Bärbel Klauke ${ }^{1}$, Volker Walhorn ${ }^{2}$, Jan Gummert ${ }^{1}$, Dario Anselmetti ${ }^{2}$ and Hendrik Milting ${ }^{1}$
${ }^{1} E$ \& $H$ Klessmann Institute for Cardiovascular Research \& Development, Heart and Diabetes Center NRW,

Ruhr-University Bochum, Bad Oeynhausen, Germany;

${ }^{2}$ Experimental Biophysics and Applied Nanoscience, Faculty of Physics and Bielefeld Institute for Biophysics and Nanoscience (BINAS),

Bielefeld University, Bielefeld, Germany;

${ }^{3}$ Physikalisch-Technische Bundesanstalt (PTB), Braunschweig, Germany

E-mail: hmilting@hdz-nrw.de

${ }^{4}$ These authors contributed equally to this work.

1 Hedberg C, Melberg A, Kuhl A, Jenne D, Oldfors A: Autosomal dominant myofibrillar myopathy with arrhythmogenic right ventricular cardiomyopathy 7 is caused by a DES mutation. Eur J Hum Genet 2012; 20: 984-985.

2 Melberg A, Oldfors A, Blomstrom-Lundqvist $\mathrm{C}$ et al: Autosomal dominant myofibrillar myopathy with arrhythmogenic right ventricular cardiomyopathy linked to chromosome 10q. Ann Neurol 1999; 46: 684-692.

3 Olivé M, Armstrong J, Miralles F et al: Phenotypic patterns of desminopathy associated with three novel mutations in the desmin gene. Neuromuscul Disord 2007; 17 443-450.

4 Brodehl A, Hedde PN, Dieding $M$ et al: Dual-color photoactivation localization microscopy of cardiomyopathy associated desmin mutants. J Biol Chem 2012; 287 16047-16057.

5 Klauke B, Kossmann S, Gaertner A et al: De novo desmin-mutation N116S is associated with arrhythmogenic right ventricular cardiomyopathy. Hum Mol Genet 2010; 19: 4595-4607.

6 Vernengo L, Chourbagi O, Panuncio A et al: Desmin myopathy with severe cardiomyopathy in a Uruguayan family due to a codon deletion in a new location within the desmin 1 A rod domain. Neuromuscul Disord 2010; 20: 178-187.

Further support for this conclusion is the finding of the same mutation segregating with desminopathy in a Spanish family. ${ }^{4}$

\section{CONFLICT OF INTEREST}

The authors declare no conflict of interest.

Carola Hedberg ${ }^{1}$, Atle Melberg ${ }^{2}$, Angelika Kuhl ${ }^{3,5}$, Dieter Jenne ${ }^{3,4}$

We appreciate the comments by Brodehl et al ${ }^{1}$ on our recent article describing a DES mutation in a family with myofibrillar myopathy and arrhythmogenic right ventricular cardiomyopathy. ${ }^{2}$ We would like to clarify that the mutation, p.P419S in the desmin gene (DES), indeed co-segregates with the disease. When we compared the muscle biopsy findings with the presence of the p.P419S DES mutation, desmin storage was found in all investigated family members with the DES mutation but not in those without the mutation. The clinical expression of the disease was highly variable within the family. The original linkage study on this family was based on combined findings from clinical examination, electromyography and muscle biopsy. ${ }^{3}$ Three of five asymptomatic individuals were incorrectly considered affected by the myopathy based on these investigations. These three individuals showed only mild and unspecific myopathic changes and no desmin storage. Whether these individuals were affected by another mild myopathy remains to be clarified. These results demonstrate diagnostic difficulties with some forms of dominantly inherited muscle diseases, as they can display a wide clinical and morphological variability even within a given family.

In conclusion, despite the report by Brodehl et $a l^{1}$, we believe that the identified desmin mutation is causative for the diseases in our family, as it segregates perfectly with desmin storage in muscle. and Anders Oldfors ${ }^{1}$
${ }^{1}$ Department of Pathology, Institute of Biomedicine, Sahlgrenska Academy at University of Gothenburg, Gothenburg, Sweden; ${ }^{2}$ Department of Neuroscience and Neurology, Uppsala University Hospital and Uppsala University, Uppsala, Sweden; ${ }^{3}$ Department of Neuroimmunology,
Max-Planck Institute of Neurobiology, Martinsried, Germany and ${ }^{4}$ Comprehensive Pneumology Center, Institute of Lung Biology and Disease (iBLD), Helmholtz Center Munich,
München-Großhadern, Germany
${ }^{5}$ Current address: Roche Diagnostics, Penzberg, Germany. E-mail: carola.hedberg@gu.se

1 Brodehl A, Dieding M, Cakar $\mathrm{H}$ et al: Functional characterization of desmin mutant p.P419S. Eur J Hum Genet 2013; 21: 589-590. myopathy with arrhythmogenic right ventricular cardiomyopathy 7 is caused by a DES mutation. Eur J Hum Genet 2012; 20: 984-985.

3 Melberg A, Oldfors A, Blomstrom-Lundqvist $\mathrm{C}$ et al: Autosomal dominant myofibrillar myopathy with arrhythmogenic right ventricular cardiomyopathy linked to chromosome 10q. Ann Neurol 1999; 46: 684-692.

4 Olive M, Armstrong J, Miralles F et al: Phenotypic patterns of desminopathy associated with three novel mutations in the desmin gene. Neuromuscul Disord 2007; 17: 443-450.
2 Hedberg C, Melberg A, Kuhl A, Jenne D, Oldfors A: Autosomal dominant myofibrillar 